\title{
Drei Strategien zur Etablierung digitaler Plattformen in der Industrie
}

Einige digitale Plattformanbieter wie Apple, Google oder Facebook realisieren im Konsumgüterbereich beispiellose Wettbewerbsvorteile. Auch produzierende Unternehmen erkennen zunehmend das Potenzial, ihren Kunden neben physischen Produkten plattformbasierte Dienstleistungen anzubieten. Dies verlangt jedoch eine deutliche Veränderung des Geschäftsmodells: Als Plattformanbieter tritt das Unternehmen nicht mehr allein als Lösungsanbieter auf, sondern bündelt das Leistungsangebot vieler Unternehmen für seine Kunden. Dieser Artikel beschreibt drei Strategien, mit denen produzierende Unternehmen ihren Weg in die Plattformökonomie ausgestalten können. Ausgehend von der Annahme, dass das Unternehmen bereits als Lösungsanbieter auftritt, stellen wir diese Strategien vor und benennen konkrete Anwendungsbeispiele, die eine Formulierung eigener Strategien inspirieren können.

\section{Hedda Lüttenberg, Daniel Beverungen, Martin Poniatowski, Dennis Kundisch und} Nancy V. Wünderlich 


\section{Plattformen erfordern die Beteiligung Dritter}

Digitale Plattformen sind aus unserem Alltag nicht mehr wegzudenken. Im Konsumgüterbereich bieten digitale Marktplätze wie Amazon oder Airbnb ihren Kunden die Möglichkeit, Angebote vieler Anbieter zu vergleichen, digitale Empfehlungsdienste zu nutzen und unterschiedlichste Artikel zu kaufen. Gleichzeitig profitieren Anbieter vom Zugang zu verschiedenen Kundengruppen und höherer Reichweite. Auch können sie digitale Dienstleistungen der Plattform nutzen, wie etwa die Zahlungsabwicklung, um den Einkauf für ihre Kunden restriktionsfrei zu gestalten.

Neben dem Konsumgüterbereich etablieren sich plattformbasierte Geschäftsmodelle zunehmend auch in anderen Sektoren wie beispielsweise im Maschinenbau zum Handeln freier Maschinenkapazitäten oder zur Vergabe von Wartungsaufträgen. Auf der Grundlage des Internet der Dinge ermöglichen Plattformen neue Interaktionen zwischen digital vernetzten Produkten sowie Anbietern und Kunden digitaler Dienstleistungen. Von 502 befragten Industrieunternehmen verfolgen $54 \%$ bereits eine Strategie zum Einsatz digitaler Plattformen und 59 \% haben in 2019 ihre Investitionen in digitale Plattformen gegenüber dem Vorjahr erhöht [1]. Das bedeutet aber auch, dass für fast die Hälfte der befragten Unternehmen digitale Plattformen noch unbekanntes Terrain sind. Fehlendes Know-how stellt aus der Sicht von 45 \% der Unternehmen das größte Hemmnis für den Einsatz digitaler Plattformen dar [1].

Entsprechend stehen viele Industrieunternehmen noch vor der Herausforderung, eine geeignete Strategie für ihren Einstieg in die Plattformökonomie zu definieren. Plattformen in einem Business-to-Business-Umfeld unterscheiden sich konzeptionell von Plattformen in einem Business-toConsumer-Umfeld und sind nochmals deutlich schwieriger zu etablieren, da Leistungen häufig komplex und individualisiert sind, während die Zahl der Kunden kleiner ist, sodass Netzwerkeffekte weniger stark ausgeprägt sein können.

Unabdingbar für die Entscheidungsfindung eines an einem Plattformgeschäftsmodell interessierten Unternehmens ist es, die Komponenten digitaler Plattformen sowie deren Wirkmechanismen im Zusammenspiel mit den Stakeholdern und ihrer Umgebungsdynamiken im Plattformökosystem [3] zu verstehen (siehe Abb. 1). Plattformanbieter können manche dieser Wirkmechanismen - wie ihre eigene Strategie, die Gestaltung der eigentlichen Plattform als Informationssystem und die Regeln zur Verwendung des Systems - selbst bestimmen. Eine Beteiligung externer Parteien und die Wirkmechanismen im größeren Wettbewerbsumfeld können sie jedoch nur indirekt beeinflussen, obwohl sie maßgebliche Erfolgsfaktoren in der Plattformökonomie darstellen. Diese indirekte Steuerbarkeit macht die erfolgreiche Etablierung einer digitalen Plattform umso schwieriger.

Unternehmen müssen hinsichtlich der Plattformökonomie zwei grundsätzliche Entscheidungen treffen [7]: Eine grundlegende Entscheidung ist, ob das Unternehmen sich überhaupt in der Plattformökonomie engagieren will. Soll keine Teilnahme erfolgen oder ist sie nicht umsetzbar, so müssen

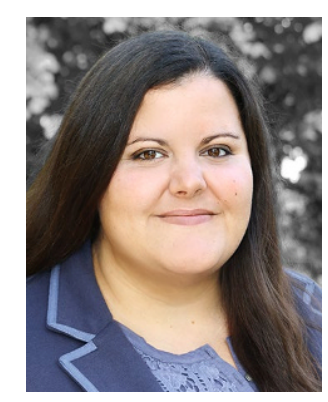

Hedda Lüttenberg ${ }^{1}$

ist wissenschaftliche Mitarbeiterin am Lehrstuhl für Wirtschaftsinformatik, insb. Betriebliche Informationssysteme der Universität Paderborn. Ihr Forschungsschwerpunkt ist das Engineering plattformbasierter Smart Service Systeme.

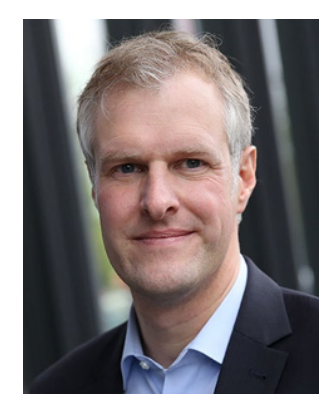

Prof. Dr. Daniel Beverungen ${ }^{1}(\square)$ ist Inhaber des Lehrstuhls für Wirtschaftsinformatik, insb. Betriebliche Informationssysteme an der Universität Paderborn. Seine Forschungsschwerpunkte umfassen die Dienstleistungsforschung, das Geschäftsprozessmanagement, die Informationsmodellierung sowie die Gestaltung und die Grenzen der Gestaltbarkeit innovativer Informationssysteme. Daniel.Beverungen@uni-paderborn.de 


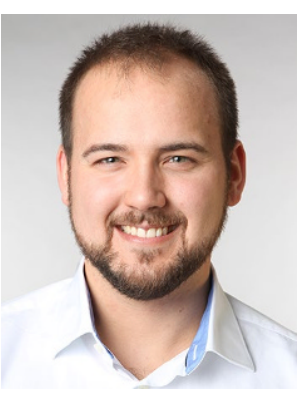

Martin Poniatowski ${ }^{1}$

ist wissenschaftlicher Mitarbeiter am Lehrstuhl für Wirtschaftsinformatik, insb. Digitale Märkte an der Universität Paderborn. Im Rahmen seiner Forschung befasst er sich mit Plattformen sowie Electronic Word of Mouth.

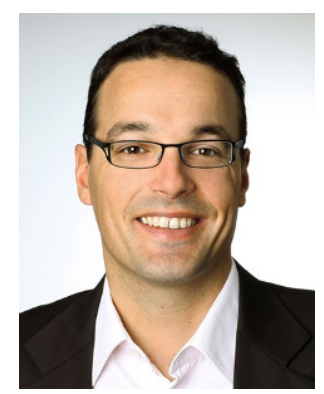

Prof. Dr. Dennis Kundisch ${ }^{1}$ ist Inhaber des Lehrstuhls für Wirtschafsinformatik, insb. Digitale Märkte an der Universität Paderborn. Weiterhin ist er Direktor des Center of Competence „Digital Business“ des Software Innovation Campus Paderborn. Seine Forschungsinteressen liegen in den Bereichen Crowdsourcing, digitale Geschäftsmodelle und Plattformen, Electronic Word of Mouth, Gamification und E-Learning. Dennis.Kundisch@uni-paderborn.de andere Möglichkeiten identifiziert werden, um sich abseits der Plattformökonomie als Produzent intelligenter Produkte oder als Lösungsanbieter zu positionieren. Fällt jedoch die Entscheidung zugunsten einer Teilnahme an der Plattformökonomie, gibt es zwei Möglichkeiten: Einerseits kann das Unternehmen als Anbieter von Sach- und Dienstleistungen auf den Plattformen anderer Unternehmen auftreten. Andererseits kann es selbst als Plattformanbieter auftreten, um Kunden und Anbieter miteinander zu vernetzen und bilaterale Transaktionen untereinander zu ermöglichen. Dabei kann das Unternehmen (schrittweise) sowohl als Plattformteilnehmer als auch als -anbieter auftreten. Diese Entscheidung muss abhängig von der bestehenden Marktsituation, den Unternehmenszielen sowie den zur Verfügung stehenden Ressourcen getroffen werden.

Langfristig ist der Erfolg einer digitalen Plattform insbesondere abhängig vom Wettbewerb, in dem sich ein Plattformanbieter strategisch positionieren muss. Mit seiner Strategie legt ein Plattformanbieter fest, in welchem Markt eine Plattform mit welchem Leistungsangebot aufgebaut werden soll. Diese Plattform ist im Kern ein Informationssystem [4], welches nach Maßgabe strategischer Überlegungen gestaltet werden muss. Die Gestaltung umfasst zum einen den technischen Entwurf der Plattform (die Infrastruktur, technische Schnittstellen etc.) und zum anderen aber auch die Governance (die Verwaltung und Steuerung) der Plattform. Hier wird beispielsweise der Grad der Offenheit festgelegt, der bestimmt, welche Nutzergruppen sich über welche Schnittstellen an der Plattform beteiligen können, sowie die Bepreisung der Leistungen. Die Governance und die technische Gestaltung der Plattform müssen dabei eng aufeinander abgestimmt werden.

Eine Plattform als Informationssystem stellt die technischen und organisatorischen Randbedingungen für die Wertschöpfung bereit. Für die Wertschöpfung selbst ist es jedoch erforderlich, dass Dritte - sowohl Kunden als auch Anbieter komplementärer Produkte und Dienstleistungen - mithilfe der Plattform miteinander interagieren. Eine Plattform muss deshalb auch ein Engagement dritter Parteien ermöglichen. Dies bedeutet, dass durch die Verwaltung und Steuerung der Plattform Anreize dafür gesetzt werden müssen, dass Kunden und andere Leistungsanbieter sich auf der Plattform engagieren und damit für sich selbst und für den Plattformanbieter einen Nutzen erzeugen. Zudem sind digitale Plattformen in ein Ökosystem eingebettet, das aufgrund einer Umgebungsdynamik nur indirekt steuerbar ist. So kann ein Plattformanbieter mithilfe von Preismodellen Anreize für Anbieter oder Kunden setzen, sich an seiner Plattform zu beteiligen. Die Entscheidung für oder gegen eine Beteiligung werden diese Akteure aber letztlich selbst treffen.

Direkte und indirekte Netzwerkeffekte spielen eine entscheidende Rolle für die Generierung von Wettbewerbsvorteilen mit Plattformen. Direkte Netzwerkeffekte bestehen innerhalb derselben Nutzergruppe: Je mehr Kunden etwa eine Plattform zum Handel mit freien Maschinenkapazitäten nutzen, desto verlässlicher wird die Qualität von Bewertungen und Rezensionen der Anbieter, wodurch zusätzliche Kunden gewonnen werden können. 
Kunden profitieren daher von der Plattformnutzung durch andere Kunden. Indirekte Netzwerkeffekte bestehen zwischen verschiedenen Nutzergruppen: Mit einer größeren Anzahl von Anbietern wird eine höhere, regionale Verfügbarkeit freier Maschinenkapazitäten erreicht, wodurch die Plattform ein attraktiverer Anlaufpunkt für Kunden wird, und umgekehrt. Daher sollten Plattformanbieter versuchen, möglichst schnell eine kritische Masse an Kunden und Anbietern für ihre Plattform zu gewinnen, um weitere Beteiligte erfolgreich einbinden zu können.

Auf der anderen Seite könnte es sowohl für Kunden als auch für Anbieter attraktiv sein, sich gleichzeitig auf mehreren Plattformen zu engagieren. Dieses sogenannte Multi-Homing kann aus Sicht eines einzelnen Plattformanbieters unerwünscht sein. Ein Multi-Homing-Verhalten kann beispielsweise durch die Verwendung proprietärer Schnittstellen eingeschränkt werden, da diese eine Beteiligung an mehreren Plattformen für Dritte aufwendiger machen. Umgekehrt birgt dies die Gefahr, größere Hürden für eine Beteiligung an der eigenen Plattform zu schaffen. Es müssen genau die richtigen Anreize gesetzt werden, die eine möglichst exklusive Beteiligung Dritter an der Plattform aus deren Sicht attraktiv machen.

Entscheidend für eine Beteiligung von Kunden und Anbietern ist zudem das Vertrauen in die Plattform und ihre Verwendung. Wichtige Aspekte umfassen etwa die glaubhafte Sicherheit und Vertraulichkeit der Daten und der Interaktionen auf der Plattform sowie das Vorhandensein effektiver Maßnahmen des Plattformbetreibers zur Qualitätssicherung der direkten Interaktionen.

Angesichts der vorgestellten Konzepte und ihrer Wechselwirkungen ist festzuhalten, dass plattformbasierte Geschäftsmodelle besonders komplex zu implementieren und zu steuern sind, da sich viele Maßnahmen des Plattformanbieters nur indirekt auf die Nutzung und den Erfolg der Plattform auswirken und durch die Einbindung externer Parteien auch ungewollte Seiteneffekte zeitigen können. Aufgrund dieser Komplexität fällt es vielen Unternehmen schwer, eigene Transformationspfade zum Plattformanbieter zu entwickeln. Es erscheint hilfreich, zunächst den Zwischenschritt der Transformation zu einem Lösungsanbieter zu gehen, der Daten über die installierte Basis seiner Produkte konsolidiert, sodass die Etablierung eines plattformbasierten Geschäftsmodells mit einem Startvorteil erfolgen kann.

\section{Smart-Service-Systeme als Zwischenschritt zum Plattformanbieter}

Mittelständische Industrieunternehmen sind häufig sehr produktzentriert ausgerichtet, sodass das Produkt und seine Eigenschaften und Funktionalitäten im Vordergrund des Leistungsportfolios stehen. Im Rahmen dieser Schwerpunktsetzung nehmen Unternehmen häufig eine eher nach innen gerichtete Perspektive ein und entwickeln ihre Leistungen basierend auf den eigenen Kompetenzen und Fähigkeiten sowie technologischen Neuerungen. Oftmals wird dabei aber eine nach außen gerichtete Perspektive vernachlässigt, die den Kunden und seine Bedürfnisse als Ausgangspunkt der Lösungs-

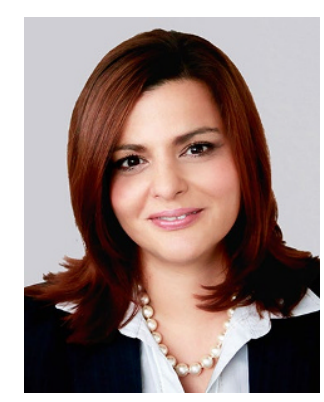

Prof. Dr. Nancy V. Wünderlich ${ }^{2}$ ist Inhaberin des Lehrstuhls für Digitale Märkte an der Technischen Universität Berlin. Sie beschäftigt sich mit der Erforschung digitaler Transformationen aus wirtschaftswissenschaftlicher Perspektive. Ihre Forschungsinteressen umfassen Managementstrategien, Geschäftsmodelle und Nutzerverhalten in digitalen Märkten, Dienstleistungsinnovationen sowie den Einsatz und die Akzeptanz neuer Technologien an der Kundenschnittstelle.

${ }^{1}$ Wirtschaftsinformatik, Universität Paderborn, Paderborn, Deutschland

${ }^{2}$ Digitale Märkte, Technische Universität Berlin, Berlin, Deutschland 


\section{Kernthese 1}

\section{Digitale Plattformen}

begründen komplexe

Geschäftsmodelle. Der

Plattformanbieter kann das

Plattformökosystem häufig

nur indirekt beeinflussen findung begreift. Statt Lösungen ausgehend von konkreten Kundenproblemen zu formulieren, fragen sich viele Unternehmen noch zu häufig, welche Leistungen sie zusätzlich zu einem bestehenden Produktportfolio anbieten können, um dessen Attraktivität zu erhöhen. Ein Plattformgeschäftsmodell verlangt jedoch einen radikalen Perspektivenwechsel: Kunden und Anbieter, nicht das Kernprodukt, müssen in den Mittelpunkt gestellt werden.

Die Etablierung von Smart-Service-Systemen kann ein geeigneter Zwischenschritt sein, um die Eintrittshürde in die Plattformökonomie zu senken. Smart-Service-Systeme basieren auf digital vernetzten physischen Produkten, die an der Schnittstelle zwischen Kunden und Anbietern platziert werden (siehe Abb. 2) und erweiterte Interaktionen ermöglichen. Hierdurch können Unternehmen profitable Leistungen anbieten, digitale Kompetenzen aufbauen und gleichzeitig Vorläuferlösungen für eine digitale Plattform entwickeln.

Ein digital vernetztes physisches Produkt (intelligentes Produkt) ermöglicht Anbietern und Kunden die Integration ihrer Ressourcen und Aktivitäten zu einer kooperativen Wertschöpfung. Bereits existierende Produkte eines Unternehmens können zu intelligenten Produkten weiterentwickelt werden oder es wird eine völlig neue Kombination aus physischen Produkten und Dienstleistungen entwickelt, um Kundenbedürfnisse bestmöglich $\mathrm{zu}$ adressieren.

In Smart-Service-Systemen erfassen intelligente Produkte Daten über ihre Nutzung, ihren Status und ihre Umgebung. Diese Daten dienen als Grundlage für die Erbringung intelligenter Dienstleistungen (Smart Service), die Kunden zusätzliche Mehrwerte bieten [5]. So können Status- und Umge-

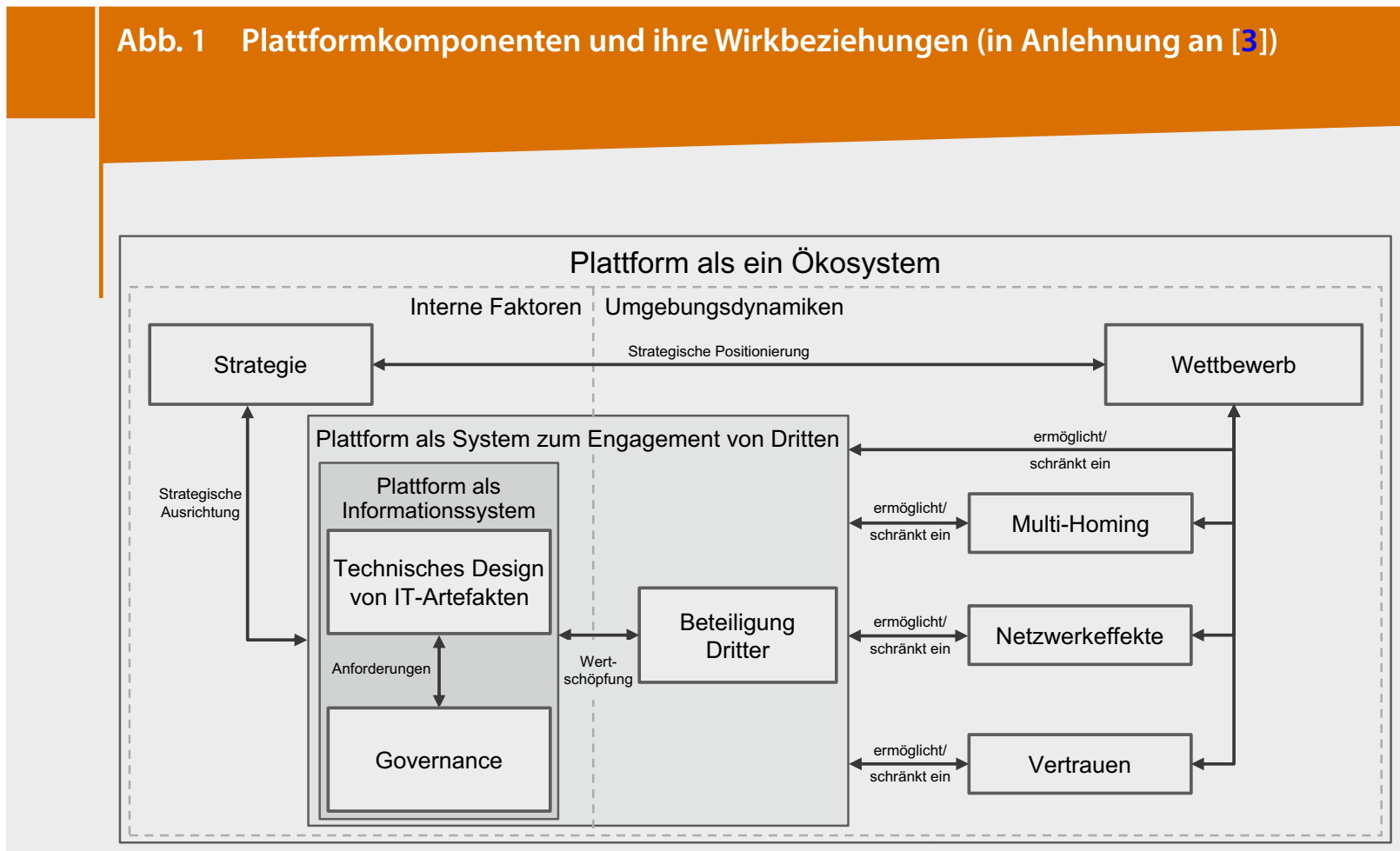


bungsdaten beispielsweise analysiert werden, um den aktuellen Zustand einer Maschine zu bewerten und vorausschauende Instandhaltungsstrategien zu ermöglichen. Einmal etabliert, können diese Daten eine wichtige Grundlage plattformbasierter Geschäftsmodelle darstellen.

Bei der Entwicklung von Smart-Service-Systemen sind jedoch auch einige Herausforderungen zu bewältigen. Insbesondere ist es erforderlich, dass sich Unternehmen von ihrem bisherigen, eher nach innen gerichteten Vorgehen lösen und stattdessen eine nach außen, auf den Kunden gerichtete Perspektive einnehmen. Eine starke Kundenorientierung ist essenziell für die erfolgreiche Dienstleistungsentwicklung und steht im Kontrast zu einem produkt- oder technologieorientierten Entwicklungsprozess. Durch eine starke Kundenorientierung bereits zu Beginn des Entwicklungsprozesses kann sichergestellt werden, dass das Smart-Service-System die Bedürfnisse des Kunden adressiert. Darüber hinaus sollte statt eines linearen Entwicklungsprozesses, wie beispielsweise Stage-Gate-Prozessen oder Phasen-Meilenstein-Prozessen, ein agiler Entwicklungsprozess bevorzugt werden (zum Beispiel nach DIN SPEC 33453 [6]), um schnell auf sich ändernde Umstände reagieren zu können. Ebenfalls sollte frühzeitig im Prozess ein erstes Geschäftsmodell entwickelt und die Zahlungsbereitschaft der Kunden überprüft werden. Zur Bewertung eines Smart-Service-Konzepts sollte neben direkten Zahlungsströmen auch der indirekte Nutzen eines Smart-ServiceSystems berücksichtigt werden. Dieser kann z. B. in einem Cross-Selling-Potenzial oder einer verbesserten Kundengewinnung bzw. -bindung bestehen. Im Rahmen einer organisationalen Transformation sind neben einer Analyse und Anpassung der bestehenden Prozesse und Organisationsstrukturen auch die erforderlichen Kompetenzen der Mitarbeiter zur Entwicklung und Erbringung des Smart Services zu identifizieren und zu schaffen.

\section{Abb. 2 Smart-Service-System (in Anlehnung an [5])}

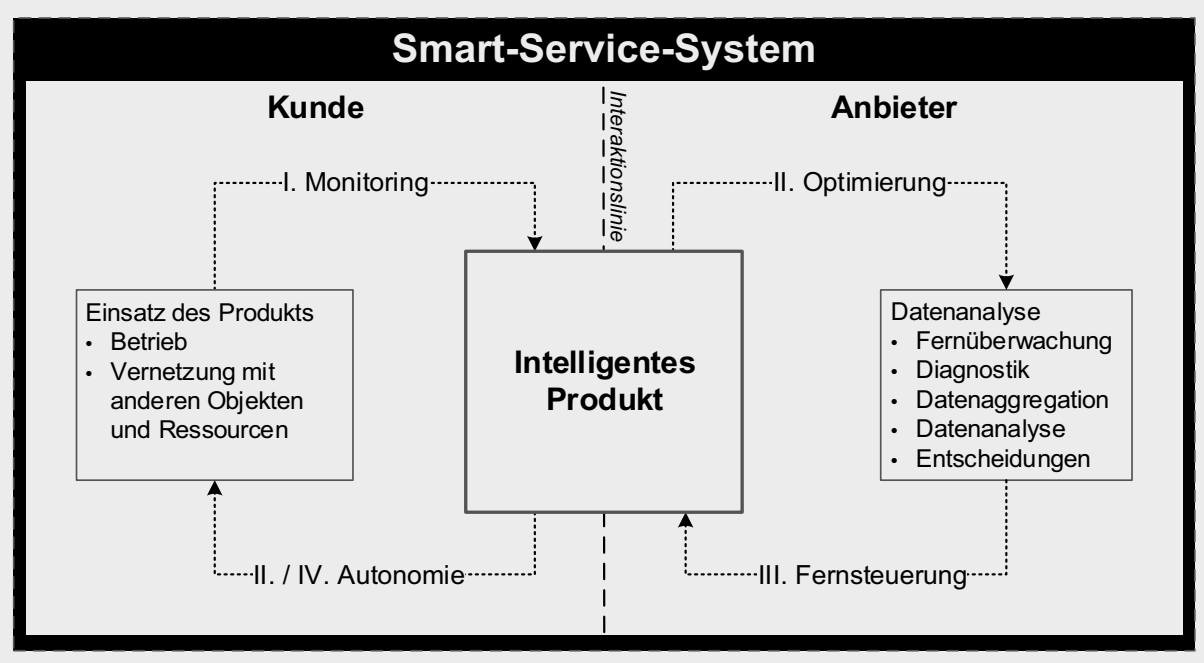




\section{Drei Strategien zur Etablierung digitaler Plattformen in der Industrie}

Sollte sich das produzierende Unternehmen dazu entscheiden, sich nicht an einer existierenden Plattform zu beteiligen, sondern selbst Plattformanbieter werden zu wollen, hat es ausgehend von bereits angebotenen Smart Services drei strategische Optionen zur Etablierung einer Plattform [7]. Diese Strategien führen zur Etablierung einer Smart-Data-Plattform, einer SmartProduct-Plattform oder einer Matching-Plattform.

\section{Smart-Data-Plattform}

Mittels einer Smart-Data-Plattform erhebt ein Plattformanbieter Daten auf der Ebene seiner intelligenten Produkte, ermöglicht Dritten jedoch nur den Zugriff auf bereinigte oder aggregierte Daten. Potenzielle Anbieter auf der Plattform können die vorverarbeiteten Daten analysieren und den Kunden darauf basierend eigene Mehrwertdienste anbieten. Es ist für sie jedoch nicht möglich, auf maschinenspezifische Daten zuzugreifen, sodass die Nutzung einzelner Maschinen nicht ausgewertet werden kann. Hierdurch bewahrt sich der Plattformanbieter einen exklusiven Zugang zu den produktbezogenen Kernbedürfnissen seiner Kunden, während Drittanbieter komplementäre Leistungen anbieten.

Ein Anwendungsbeispiel für eine Smart-Data-Plattform stellt die Plattform „DENIOS CONNECT“ der DENIOS AG aus Bad Oeynhausen dar.

\section{Kernthese 2}

\section{Aufbauend auf digital vernetz- ten physischen Produkten stellen Smart-Service-Systeme vielfach einen sinnvollen Zwischenschritt auf dem Weg zum Plattformanbieter dar} DENIOS produziert und vertreibt Raumsysteme zur sicheren und gesetzeskonformen Gefahrstofflagerung. Um Smart Services anbieten zu können, wurden diese Raumsysteme weiterentwickelt und mit Sensorik und weiteren elektronischen Komponenten ausgestattet. Auf Basis eines Prototyps zum intelligenten Lagermanagement erfasst und analysiert die Plattform Daten über die Art der gelagerten Gefahrstoffe, die Ein- und Auslagerung der Gefahrstoffe sowie die Stellplatzbelegung. Der Kunde profitiert unter anderem davon, dass Informationen zu den Gefahrstoffen (wie beispielsweise benötigte Schutzausrüstung) direkt vor Ort verfügbar sind und durch das System geprüft werden. Zudem unterstützen intelligente Regalsysteme die gesetzeskonforme Lagerung der Gefahrstoffe und erlauben, dass die Auslastung des Lagers jederzeit überprüft werden kann. Wie an diesem Beispiel deutlich wird, hätte DENIOS Zugriff auf die Daten jedes intelligenten Produktes, in diesem Fall eines jeden intelligenten Raumsystems, und könnte diese Daten verwenden, um für den Kunden Smart Services zu erbringen.

Gleichzeitig könnte DENIOS aggregierte Daten Dritten zur Verfügung stellen, die darauf basierend Mehrwertdienste anbieten können. Ein Beispiel können externe Dozenten sein, die Schulungen im Umgang mit Gefahrstoffen auf die Kombination und Mengen der gelagerten Gefahrstoffe zuschneiden und so für den Kunden personalisieren können. Auch kann ein Benchmarking von Kunden basierend auf der Kombination von Gefahrstoffen und deren Handling etabliert werden. Während DENIOS seinen Kunden den Benchmark zur eigenen Bewertung verfügbar machen kann, könnte DENIOS auch Dritten Zugriff auf diese aggregierten Daten gewähren. So wäre es 
vorstellbar, dass Versicherer Interesse an einem Zugriff auf die Daten haben, um den Kunden individuelle Angebote unterbreiten zu können.

Für die Umsetzung einer Smart-Data-Plattform muss ein Plattformanbieter zunächst analysieren, welche Art von aggregierten Daten aus seinem SmartService-System für Dritte interessant sein könnten. Da die Daten nicht auf Maschinenebene analysiert werden können, müssen sich auch basierend auf den aggregierten und bereinigten Daten Mehrwertdienstleistungen anbieten lassen können. Vor allem in Nischen bzw. bei sehr spezifischen Daten, wie in unserem Anwendungsbeispiel zur Gefahrstofflagerung, können auch aggregierte Daten interessante Einblicke liefern. Bei aggregierten Daten, die nicht einem bestimmten Kontext zugeordnet werden können (zum Beispiel einer Branche, einem Anwendungsgebiet, einer Fertigungsart), ist die Interpretierbarkeit dieser Daten eingeschränkt, wodurch auch deren Verwendbarkeit für Mehrwertdienste erschwert ist. Wurden geeignete Daten identifiziert, die auf einer Plattform angeboten werden können, sollte geprüft werden, ob bereits Plattformen existieren, die vergleichbare Daten und Dienstleistungen bereitstellen. Insbesondere sollte auch geprüft werden, ob vergleichbare Daten auf Plattformen verfügbar sind, die auf den Handel von Daten spezialisiert sind. Es ist sicherzustellen, dass Anbieter und Kunden durch die zusätzlichen Leistungen einen Mehrwert erzielen, an dem der Plattformbetreiber partizipiert.

\section{Smart-Product-Plattform}

Im Vergleich zur Smart-Data-Plattform ist eine Smart-Product-Plattform dadurch gekennzeichnet, dass Dritte auf Daten auf Maschinenebene zugreifen können, um Mehrwertdienste anzubieten. Der Plattformanbieter kann jedoch durchaus Einschränkungen in den für Dritte verfügbaren Daten vornehmen, sodass ausschließlich er selbst auf bestimmte Daten zugreifen und sich dadurch einen Wettbewerbsvorteil sichern kann.

Ein Beispiel für eine Smart-Product-Plattform ist die WAGO Cloud der WAGO Kontakttechnik GmbH \& Co. KG aus Minden. WAGO stellt Komponenten für die elektrische Verbindungstechnik sowie elektronische Komponenten für die dezentrale Automatisierungstechnik her. Einige dieser Komponenten ermöglichen es, Daten von Maschinen und Anlagen, in denen sie eingesetzt werden, zentral zu sammeln und zu analysieren. WAGO gewährt den Kunden über die WAGO Cloud Zugriff auf die angebundenen Komponenten sowie die Möglichkeit, an diesen Komponenten per Fernzugriff Einstellungen vorzunehmen. Darüber hinaus können sich die Kunden über personalisierbare Dashboards die erfassten Daten, wie beispielsweise Füllstände oder Energieverbräuche, aufbereitet anzeigen lassen oder Alarme bei Eintritt von bestimmten Zuständen definieren.

WAGO könnte nun die Plattform für ausgewählte externe Partner öffnen, die den Kunden auf den Daten basierende Mehrwertdienste anbieten. Ein Beispiel könnten Beratungsunternehmen sein, die produzierende Unternehmen im Hinblick auf Energieeinsparpotenziale oder Prozessoptimierungen beraten. Durch den maschinenspezifischen Zugriff auf Daten können sie auf Basis von maschinellem Lernen Fehlerquellen identifizieren und gezielt Ver- 
besserungsmaßnahmen ableiten. Auch Prognosen, beispielsweise zu Energieverbräuchen oder produzierten Stückzahlen, sowie Simulationen von unterschiedlichen Szenarien sind für den Kunden wertvolle Leistungen, die durch Dritte angeboten werden können. Werden auf der Smart-ProductPlattform zunächst ausgewählte Partner zugelassen, kann der Anbieter - hier WAGO - zunächst selbst das Leistungsspektrum der Drittparteien steuern und so gezielt Leistungsangebote auswählen, die sie selbst nicht erbringen wollen. So schaffen diese Leistungsangebote einen Mehrwert für den Kunden gegenüber dem eigenen Angebotsportfolio, unterliegen aber noch vergleichsweise stark der Kontrolle und Steuerung durch den Plattformanbieter.

Für die Umsetzung einer Smart-Product-Plattform muss ein Plattformanbieter sicherstellen, dass die Kunden bereit sind, Daten auf Maschinenebene für Drittanbieter auf der Plattform verfügbar zu machen. Im Vergleich zur Smart-Data-Plattform, auf der durch die Aggregation bzw. Bereinigung der Daten eine gewisse Anonymität und Abstraktion vom Kerngeschäft erreicht wird, entstehen bei den Kunden von Smart-Product-Plattformen oft Bedenken in Bezug auf die Datensicherheit und ob aus den verfügbaren Daten Rückschlüsse auf Firmengeheimnisse oder essenzielles Wissen hergestellt werden könnten. Der Plattformanbieter muss also sicherstellen, dass die Daten seiner Kunden entsprechend geschützt werden, Dritte aber gleichwohl in ausreichendem Maße auf Daten zugreifen können, um ihre Mehrwertdienste anzubieten.

\section{Matching-Plattform}

Eine Matching-Plattform ist im Vergleich zu den beiden vorgenannten Plattformtypen dadurch gekennzeichnet, dass sie keine direkte Verbindung zum intelligenten Produkt und den dort erhobenen Daten hat. Das Leistungsangebot der Plattform besteht vielmehr darin, eine Kundennachfrage mit dem Leistungsangebot dritter Parteien zusammenzubringen.

Zur Veranschaulichung der Matching-Plattform wird noch einmal die DENIOS AG herangezogen. Als Basis für den Einstieg in die Plattformökonomie bietet DENIOS seinen Kunden einen intelligenten Condition-Monitoring-Service für ihre vernetzten Raumsysteme an. Die Kunden können über die Plattform auf ein Dashboard zugreifen, auf dem jederzeit der aktuelle Status des Raumsystems, aber auch historische Werte einsehbar sind. Relevant ist hier zum Beispiel die Temperatur im Lager, die bei einigen Gefahrstoffen immer in einem definierten Wertebereich liegen muss. Auch ob die Lüftung funktionsfähig ist oder die Tür offen steht, ist eine relevante Information für die Kunden. Als Bestandteil des Condition-MonitoringService können Kunden im Bedarfsfall direkt über die Plattform eine Wartungsanfrage an DENIOS stellen.

Hier eröffnet sich nun für DENIOS das Potenzial, die Plattform für qualifizierte Partner zu öffnen. Bei einer Wartungsanfrage über die Plattform findet dann ein Matching zwischen der Anfrage und einem in der Nähe befindlichen, verfügbaren und qualifizierten Dienstleister statt, der die Wartung zügig durchführen kann. Gerade wenn lokal keine eigenen Servicetechniker verfügbar sind oder dem Kunden ein Leistungsversprechen in Bezug 
auf eine festgelegte Reaktionszeit gegeben wurde, ist es unter Umständen erforderlich, weiteren Partnern Zugriff auf die Plattform zu gewähren, um die eingehenden Anfragen auch bedienen zu können. Dies ist insbesondere bei steigender Auftragszahl der Fall.

Für die Umsetzung einer Matching-Plattform muss ein Plattformanbieter eine wertschaffende Vermittlerrolle innerhalb eines Wertschöpfungsnetzwerks einnehmen. Plattformanbieter können davon profitieren, dass externe Anbieter komplementäre Leistungen zu ihrem eigenen Kerngeschäft erbringen. Da eine Matching-Plattform jedoch keinen direkten Bezug zum intelligenten Produkt oder den dort generierten Daten erfordert, könnten mittelfristig jedoch konkurrierende Plattformen mit einem stärkeren Produktbezug entstehen. In diesem Fall sollte der Plattformanbieter erwägen, ein Multi-Homing zuzulassen. Darüber hinaus besteht bei Matching-Plattformen eine erhöhte Gefahr für das Entstehen von Meta-Plattformen, die Angebote mehrerer ähnlicher Plattformen bündeln.

\section{Plattformbasierte Geschäftsmodelle sind lukrativ, aber riskant}

Die Entscheidung, eine digitale Plattform zu etablieren, ist trotz der hier aufgezeigten Optionen keineswegs ohne Risiko. Unter anderem aufgrund des sog. „Überlebensirrtums“ [8] kann schnell der Eindruck entstehen, dass es jedes Unternehmen schafft, eine erfolgreiche Plattform zu etablieren. Tatsächlich sind jedoch Anbieter digitaler Plattformen häufig gescheitert und viele Plattformen sind bis heute nicht profitabel.

Eine Vielzahl an Herausforderungen (beispielsweise: richtiges Verhältnis zwischen Offenheit und Kontrolle, Incentivierung Dritter etc. [9, 10]) ist auf dem Weg zur einer erfolgreichen, nachhaltigen sowie vor allem profitablen Plattform zu bewältigen. Zu den größten Herausforderungen bei der Etablierung einer Plattform zählt insbesondere die Bewältigung des Henne-EiProblems (oder auch Kaltstart-Problem), dessen Lösung für die Etablierung von Netzwerkeffekten existenziell ist [2]. Denn eine Plattform funktioniert nur, solange es eine kritische Masse an Teilnehmern gibt und die Plattform ein bestimmtes Aktivitätslevel aufrechterhalten kann. Der Plattformanbieter muss externen Beteiligten daher ein attraktives Angebot zur Beteiligung an der Plattform machen, das zunächst auch ohne das Vorhandensein der Netzwerkeffekte greift. Mit zunehmender Attraktivität der Plattform entstehen dann Netzwerkeffekte, aufgrund derer der Plattformanbieter ein überlegenes Geschäftsmodell realisieren kann. Bis dies jedoch geschieht, könnte die Plattform - wie viele Anwendungsbeispiele im Konsumgüterbereich belegen - defizitär sein. Plattformanbieter benötigen daher einen langen Atem.

Fest im Markt etablierte Unternehmen haben hier jedoch einen Vorteil: In den meisten Fällen besitzen sie bereits eine Vielzahl an sehr gut etablierten Partnerschaften entlang der Wertschöpfungskette und können diese nutzen, um ihre Geschäftspartner als Leistungsanbieter für ihre Plattform zu gewinnen. Die Teilnahme an der Plattform hätte auch für die Partner den Vorteil, bereits frühzeitig Teil der Plattform zu sein und somit eine größere Kunden-

\section{Zusammenfassung}

- Smart-Data-Plattformen stellen aggregierte, bereinigte Daten bereit

- Smart-Product-Plattform ermöglichen den Zugriff auf maschinenspezifische Daten

- Matching-Plattformen etablieren digitale Marktplätze ohne direkten Produktbezug 


\section{Handllungsempfehlungen}

- Smart-Service-Systeme als Vorstufe digitaler Plattformen etablieren

- Daten aus digital vernetzten Produkten konzeptionell definieren und sammeln

- Lösungsbausteine zur Plattform weiterentwickeln und Handlungsumfeld bedenken reichweite zu erlangen. So wäre die Plattform bereits bei ihrer Etablierung unmittelbar nutzenstiftend für die Anbieter und ihre existierende Kundenbasis. Auf dieser Grundlage würde es daher leichter fallen, zusätzliche Leistungsanbieter und Kunden zu einer Mitwirkung auf der Plattform zu bewegen.

Bei der Entwicklung einer Strategie ist zudem zu beachten, dass in der Plattformökonomie häufig Winner-takes-all-Marktkonstellationen entstehen - im Vorteil ist dabei häufig die globale oder, im Sinne der angebotenen Kundenlösung, allgemeiner aufgestellte Plattform. Unternehmen, die als Plattformanbieter auftreten wollen, müssen für sich daher eine Wettbewerbsposition etablieren, die langfristig, beispielsweise auch gegen die Anbieter übergeordneter Kundenlösungen, verteidigungsfähig ist. Andernfalls werden sie zwar das initiale Investment getätigt haben, jedoch kaum von den Vorteilen einer dominanten Plattform profitieren.

Unsere Empfehlung ist, dass produzierende Unternehmen den Weg zum Plattformanbieter iterativ beschreiten sollten. Insbesondere die Etablierung von Smart-Service-Systemen kann neue Marktpotenziale erschließen, gleichzeitig aber auch eine vielversprechende Ausgangsposition zur Einführung einer digitalen Plattform darstellen.

Förderung. Die in diesem Artikel beschriebenen Forschungsergebnisse wurden im Projekt „Digital Busines““ im Rahmen des Technologienetzwerks it’s OWL (Fördernummer: 005-1807-0107) entwickelt. Wir danken dem Ministerium für Wirtschaft, Innovation, Digitalisierung und Energie des Landes Nordrhein-Westfalen und dem Projektträger Jülich (PTJ) für die Unterstützung. Des Weiteren wurde diese Arbeit zum Teil von der Deutschen Forschungsgesellschaft (DFG) im Zuge des Sonderforschungsbereichs „OnThe-Fly Computing“ (GZ: SFB 901/3) mit der Projektnummer 160364472 unterstützt.

Funding. Open Access funding enabled and organized by Projekt DEAL.

Open Access Dieser Artikel wird unter der Creative Commons Namensnennung 4.0 International Lizenz veröffentlicht, welche die Nutzung, Vervielfältigung, Bearbeitung, Verbreitung und Wiedergabe in jeglichem Medium und Format erlaubt, sofern Sie den/die ursprünglichen Autor(en) und die Quelle ordnungsgemäß nennen, einen Link zur Creative Commons Lizenz beifügen und angeben, ob Änderungen vorgenommen wurden.

Die in diesem Artikel enthaltenen Bilder und sonstiges Drittmaterial unterliegen ebenfalls der genannten Creative Commons Lizenz, sofern sich aus der Abbildungslegende nichts anderes ergibt. Sofern das betreffende Material nicht unter der genannten Creative Commons Lizenz steht und die betreffende Handlung nicht nach gesetzlichen Vorschriften erlaubt ist, ist für die oben aufgeführten Weiterverwendungen des Materials die Einwilligung des jeweiligen Rechteinhabers einzuholen.

Weitere Details zur Lizenz entnehmen Sie bitte der Lizenzinformation auf http://creativecommons.org/licenses/by/4.0/deed.de. 


\section{Literatur}

[1] Bitkom Research (2020). Digitale Plattformen. Chartbericht. Berlin: Bitkom Research.

[2] Stummer, C., Kundisch, D., \& Decker, R. (2018). Platform launch strategies. Bus Inf Syst Eng, 60, 167-173.

[3] Poniatowski, M., Lüttenberg, H., Beverungen, D., \& Kundisch, D. (2021). Three layers of abstraction-A conceptual framework for theorizing digital multi-sided platforms. In Information systems and e-business management. Special issue on platform business models and platform strategies.

[4] de Reuver, M., Sørensen, C., \& Basole, R. C. (2018). The digital platform: a research agenda. Journal of Information Technology, 33, 124-135.

[5] Beverungen, D., Müller, O., Matzner, M., Mendling, J., \& Vom Brocke, J. (2019). Conceptualizing smart service systems. Electronic Markets, 29, 7-18.

[6] DIN: DIN SPEC 33453:2019-09 (2019): Entwicklung Digitaler Dienstleistungssysteme. Beuth Verlag, Berlin

[7] Beverungen, D., Kundisch, D., \& Wünderlich, N. (2020). Transforming into a platform provider: strategic options for industrial smart service providers. JOSM. https://doi.org/10.1108/JOSM-03-2020-0066.

[8] Glaser, C. (2019). Überlebensirrtum. In C. Glaser (Hrsg.), Risiko im Management. 100 Fehler, Irrtümer, Verzerrungen und wie man sie vermeidet (S. 125-128). Wiesbaden: Springer.

[9] Van Alstyne, M. W., Parker, G., \& Choudary, S. P. (2016). 6 reasons platforms fail. Harvard business review, 31, 2-6.

[10] Van Alstyne, M. W., Parker, G., \& Choudary, S. P. (2016). Pipelines, platforms, and the new rules of strategy. Harvard business review, 94, 54-62. 